\title{
Two canine CD1a proteins are differentially expressed in skin
}

\author{
Frank A. Looringh van Beeck • Dirk M. Zajonc • \\ Peter F. Moore • Yvette M. Schlotter • Femke Broere • \\ Victor P. M. G. Rutten • Ton Willemse • \\ Ildiko Van Rhijn
}

Received: 21 February 2008 / Accepted: 7 April 2008 / Published online: 17 May 2008

(C) The Author(s) 2008

\begin{abstract}
Lipid antigens are presented to T cells by the CD1 family of proteins. In this study, we characterize the complete $\operatorname{dog}$ (Canis familiaris) CD1 locus, which is located on chromosome 38. The canine locus contains eight CD1A genes (canCD1A), of which five are pseudogenes, one canCD1B, one canCD1C, one canCD1D, and one canCD1E gene. In vivo expression of canine $\mathrm{CD} 1$ proteins was shown for canCD1a6, canCD1a8, and canCD1b, using a panel of anti-CD1 monoclonal antibodies (mAbs). CanCD1a6 and canCD1a8 are recognized by two distinct mAbs. Further-
\end{abstract}

F. A. Looringh van Beeck · F. Broere • V. P. M. G. Rutten •

T. Willemse $\cdot$ I. Van Rhijn $(\bowtie)$

Department of Infectious Diseases and Immunology,

Faculty of Veterinary Medicine, University Utrecht,

Yalelaan 1,

3584 CL Utrecht, The Netherlands

e-mail: i.vanrhijn@uu.nl

D. M. Zajonc

Division of Cell Biology,

La Jolla Institute for Allergy and Immunology,

9420 Athena Circle,

La Jolla, CA 92037, USA

P. F. Moore

Department of Pathology, Microbiology and Immunology,

School of Veterinary Medicine, University of California at Davis,

Davis, CA 95616, USA

Y. M. Schlotter · T. Willemse

Department of Clinical Sciences of Companion Animals,

Faculty of Veterinary Medicine, University Utrecht,

Yalelaan 108, 3584 CM Utrecht,

The Netherlands

V. P. M. G. Rutten

Department of Tropical Veterinary Diseases, Faculty of Veterinary

Science, University of Pretoria,

Private Bag X04,

Onderstepoort 0110, South Africa more, we show differential transcription of the three canCD1A genes in canine tissues. In canine skin, the transcription level of canCD1A8 was higher than that of canCD1A6, and no transcription of canCD1A2 was detected. Based on protein modeling and protein sequence alignment, we predict that both canine CD1a proteins can bind different glycolipids in their groove. Besides differences in ectodomain structure, we observed the unique presence of three types of cytoplasmic tails encoded by canCD1A genes. cDNA sequencing and expressed sequence tag sequences confirmed the existence of a short, human CD1a-like cytoplasmic tail of four amino acids, of an intermediate length form of 15 amino acids, and of a long form of 31 amino acids.

Keywords Skin immunology $\cdot$ CD1 antigens ·

Animal models

\section{Introduction}

Antigen presenting molecules play an important role in the adaptive immune system. The well-known major histocompatibility complex (MHC) class I and class II antigenpresenting molecules have been found in all jawed vertebrates studied so far. In mammals and birds, an additional antigen presenting lineage has been identified: the nonpolymorphic CD1 family of glycoproteins consisting of CD1a, CD1b, CD1c, CD1d, and CD1e. The remarkable difference between $\mathrm{CD} 1$ and $\mathrm{MHC}$ is that $\mathrm{CD} 1$ molecules present foreign and self-lipid antigens, rather than peptides to T cells (Beckman et al. 1994; Beckman et al. 1996; Spada et al. 1998; De Libero and Mori 2005; Barral and Brenner 2007). It has been shown that different classes of lipid antigens are presented by different $\mathrm{CD} 1$ isoforms. The small binding groove of CD1a allows the binding of mycobacterial lipopeptides and 
sulfatide sphingolipid (Shamshiev et al. 2002; Moody et al. 2004; Zajonc et al. 2005), whereas CD1b has the largest binding groove of all $\mathrm{CD} 1$ isoforms and is capable to bind lipid antigens with much longer alkyl chains, such as mycolyl lipids (Beckman et al. 1994; Moody et al. 1997; Ernst et al. 1998; Niazi et al. 2001). Not only the size and shape of the CD1 groove structure but also intracellular trafficking determines which lipid antigens are presented by which CD1 isoform. Human CD1a has been shown to traffic to early endosomes were it samples lipid antigens and translocates back to the cell surface. Human CD1b, CD1c, and CD1d traffic to lysosomes and/or late endosomal compartments within the cell where they encounter other types of lipid antigens (Sugita et al. 1996; Jackman et al. 1998; Sugita et al. 1999; Briken et al. 2000; Brigl and Brenner 2004; Sugita et al. 2007).

Another difference between CD1 isoforms is their expression pattern and the type of T cell they activate. Group $1 \mathrm{CD} 1$ molecules (CD1a, CD1b, and CD1c) are expressed on professional antigen-presenting cells. Human Langerhans cells in the skin are known for their high expression of CD1a, while dermal dendritic cells express CD1b and CD1c (Dougan et al. 2007). It has been shown that group 1 CD1 molecules present mycobacterial antigens to $\mathrm{T}$ cells (Beckman et al. 1994; Moody et al. 2000; Pena-Cruz et al. 2003; Moody et al. 2004). Group 2 CD1 molecules (CD1d) are expressed on several types of antigen-presenting cells but are also present on a wide range of nonhematopoietic cells. CD1d is the restricting element that selects and activates invariant natural killer T cells (Bendelac et al. 1995; Chen et al. 1997; Kawano et al. 1997; Gumperz et al. 2002).

The human CD1 locus contains one gene for each of the different CD1 isoforms (Calabi and Milstein 1986; Shiina et al. 2001). However, as a result of CD1 gene duplication and deletion during mammalian evolution, the number of CD1 molecules and their genes is different in other species. For example, the guinea pig CD1 locus consists of four $\mathrm{CD} 1 \mathrm{~B}$ genes and three CD1C genes. It is thought that the loss of CD1 isoform diversity and their specific function is at least partly recovered by gene duplication and neofunctionalization of CD1 molecules (Dascher et al. 1999).

In this study, the complete dog CD1 locus is characterized. We show the differential expression of CD1A genes in canine skin and the unique in vivo expression of two distinct CD1a proteins.

\section{Materials and methods}

In silico identification of canine $\mathrm{CD} 1$ genes

Blast searches in the canine genome (Broad Institute of MIT/Harvard, CanFam 2.0 version 46.2d; coverage 7.5), available at www.ensembl.org/Canis_familiaris, were performed with $\alpha 1$ and $\alpha 2$ domain nucleotide sequences of all human CD1 isoforms. To compare canine CD1A genes with other mammalian CD1A genes, we used the known CD1A genes in GenBank of human (NM001763), rabbit (AF276977 and AF276978), pig (AF059492), and cattle (DQ192541). To identify cat CD1A genes, we performed a blast search with the human $\alpha 1$ and $\alpha 2$ domain nucleotide sequences of human CD1A in the feline genome (Agencourt Bioscience, CAT version 46.1b; coverage 2). Blast searches for expressed sequence tag (EST) sequences were performed at the National Center for Biotechnology Information website (http://www.ncbi.nlm.nih.gov/blast/Blast.cgi).

\section{Canine tissue collection and cDNA synthesis}

Tissue samples were obtained from healthy adult dogs (Beagles). Peripheral blood mononuclear cells (PBMCs) were isolated from dog blood by standard Ficoll-Hypaque gradient centrifugation. Skin samples were processed using a Biopulverizer and homogenized using a mini-Turrax (IKA). Intestine tissue (duodenum, jejunum, and colon) was washed and incubated in phosphate-buffered saline (PBS) with $0.75 \mathrm{mg} / \mathrm{ml}$ ethylenediamine tetraacetic acid, $5 \%$ fetal calf serum (FCS), and $50 \mu \mathrm{g} / \mathrm{ml}$ gentamicin at $37^{\circ} \mathrm{C}$ for $1 \mathrm{~h}$ to obtain intestinal cells. Single-cell suspensions were freshly prepared from the liver, spleen, lymph node, and thymus. Canine tissue was collected according to the regulations of the Animal Ethical Committee of the University of Utrecht, The Netherlands (protocol number 2007.II.06.152). From all collected tissues, ribonucleic acid (RNA) was isolated using the RNeasy kit (Qiagen) followed by complementary deoxyribonucleic acid (cDNA) synthesis with Multiscribe reverse transcriptase (Applied Biosystems).

PCR, cloning, and sequence analysis

Polymerase chain reactions (PCRs) were performed with Pfu Turbo polymerase (Stratagene) according to the protocol of the manufacturer under the following cycling conditions: an initial denaturation of $7 \mathrm{~min}$ at $95^{\circ} \mathrm{C}$, followed by 40 cycles of $15 \mathrm{~s}$ at $95^{\circ} \mathrm{C}, 45 \mathrm{~s}$ at a primerspecific annealing temperature, $30 \mathrm{~s}$ at $72^{\circ} \mathrm{C}$, followed by a final elongation step of $5 \mathrm{~min}$ at $72^{\circ} \mathrm{C}$. PCR for full-length $\mathrm{CD} 1$ genes was performed using thymus cDNA as a template and the following primers: canCD1A6 forward 5'-GCAAGAGAAAGACATCTGCAAACACG and reverse 5'-CCCTGGACTGACCTCAAGGCA at annealing temperature $(\mathrm{Ta})$ of $61^{\circ} \mathrm{C}$; canCD1A8 forward $5^{\prime}$-GCAA GAGAAAGACATCTGCAAACACG and reverse 5'CACRGGGAGTGGCCAGGAC at Ta of $61^{\circ} \mathrm{C}$; canCD1B forward 5'-CAGCCCACTGTCCGGGGAG and reverse 5'AAGAAGAGTTCATGAGATGGCGAGGG at Ta of $61^{\circ} \mathrm{C}$; 
canCD1C forward 5'-GGGAGCGGGGAAGCATCTGC and reverse $5^{\prime}$-GGGAGGCATGGTGACGAAGC at Ta of $59^{\circ} \mathrm{C}$. The CD1D gene was not amplified in full length due to an unknown sequence upstream from the $\alpha 2$ domain sequence; therefore, canCD1D forward was designed in the $\alpha 2$ domain 5'-GATCCTGAGTTTCCAAGGGTCTCAC and reverse 5 '-GCTGAGGGTGAAGAAAGGCTGC in the $3^{\prime}$ untranslated region at $\mathrm{Ta}$ of $65^{\circ} \mathrm{C}$. To determine the messenger RNA expression of the different CD1A genes in dog tissue, gene-specific forward primers and a general reverse primer were designed: canCD1A2 forward in $\alpha 2$ domain 5'-AGCTGCGCATTGGAGAACCTTTC; canCD1A6 forward in $\alpha 1$ domain 5'-GGACAGTGA CTCTGGCACTTTCTTG; canCD1A8 forward in $\alpha 1$ domain 5'-CATACACCATCCGATCCCCTTCC; canCD1A general reverse primer in $\alpha 3$ domain $5^{\prime}$-AGACTGCT GTGTCTCACACGGC at a Ta of $59^{\circ} \mathrm{C}$. Gel electrophoresis $(10 \%$ agarose gel) was performed for $1 \mathrm{~h}$ at $100 \mathrm{~V}$. After PCR, full-length CD1 PCR products were isolated from the gel. Products were ligated in a pCR4BluntTOPO vector (Invitrogen) to transform One Shot TOP10 cells (Invitrogen). Vector DNA of single colonies was sequenced by BaseClear (Leiden, The Netherlands). The obtained sequences were compared to the canine CD1 sequences obtained from the ensemble database (www. ensembl.org/Canis_familiaris). The ExPASy Translate Tool was used (www.expasy.ch/tools/dna) for translation into amino acids. All alignments were performed with ClustalW (align.genome.jp).

Monoclonal antibodies and staining procedures

The expression of CD1 by canine thymocytes, 030-D histiocytosis cell line (Levy et al. 1995), and 293T cells transfected with canCD1 was measured using a FACScalibur flow cytometer (Becton Dickinson). Transfection of 293 T was performed with full-length CD1 gene sequences in pcDNA3.1+ vector (Invitrogen) using FuGENE6 (Roche) as transfection reagent. Anti-canine CD1 monoclonal antibodies (mAbs) CA13.9H11 (IgG1), CA9.AG5 (IgG1), anti-feline CD1a Fe1.5F4 (IgG1), and anti-feline $\mathrm{CD} 1 \mathrm{c}$ Fe5.5C1 (IgG1) were provided by Dr. P.M. Moore (School of Veterinary Medicine, University of California, Davis, CA, USA). Anti-human CD1a (OKT6; IgG1), CD1b (BCD1b3; IgG1), and CD1c (F10/21A3; IgG1) were provided by Dr. D.B. Moody (Brigham and Women's Hospital and Harvard Medical School, Boston, MA, USA). Anti-human CD1a (WM-35; IgG2b) was obtained from ImmunoTools. Anti-bovine CD1 CC20 (IgG2a) was provided by Dr. C.J. Howard (Institute for Animal Health, Compton, UK); CC14 (IgG1) was provided by Dr. J.C. Hope (Institute for Animal Health Compton, UK); 20.27 SBU-T6 (IgG1) was obtained from the European Collection of Cell Cultures. Goat anti-mouse fluorescein isothiocyanate (GAM-FITC) was used as secondary antibody. Primary antibodies were diluted in PBS with $0.1 \%$ sodium azide and 2\% FCS. Canine thymocytes, 030-D cell lines, and CD1-transfected 293T cells were incubated with primary antibody for $30 \mathrm{~min}$ at $4^{\circ} \mathrm{C}$, followed by incubation with GAM-FITC (BD Biosciences) for $30 \mathrm{~min}$ at $4^{\circ} \mathrm{C}$.

\section{Protein modeling}

Canine CD1a protein models were generated using the Swiss Model Server with human CD1a (PDB code 1ONQ) as a template. The model was visualized using PyMol (pymol.sourceforge.net). The program APBS (Baker et al. 2001) was used to calculate the electrostatic surface potentials with electronegative in red and electropositive in blue $(-30$ to $+30 \mathrm{kT} / \mathrm{e})$.

\section{Results}

\section{Characterization of the canine CD1 locus}

The canine CD1 (canCD1) genes are located close to the telomeric end of chromosome 38 between 26.30 and 26.55 Mbp. The boundaries of the canCD1 locus are formed by the CD1-unrelated Kirrel and olfactory receptor gene group of which OR10T2 was the first gene adjacent to the CD1 locus. The canCD1 locus contains six gaps of 61578 nucleotides, which are located between the $\mathrm{CD} 1$ genes, and one gap of 2,852 nucleotides that lies between base pairs $26,503,057$ and $26,505,908$ of which $75 \%$ of the nucleotide sequence was present in a draft sequence deposited by the Broad Institute (AC183576). The canCD1 locus consists of the following genes from the telomeric end to the centromere: one canCD1D gene, eight canCD1A genes, one canCD1C, one canCD1B, and one canCD1E gene (Fig. 1). The canine $\mathrm{CD} 1$ genes have the same orientation as human $\mathrm{CD} 1$ genes except for CD1B, which in the human CD1 locus has an opposite orientation compared to the other human CD1 isoforms.

Of the eight CD1A genes, three CD1A genes (canCD1A2, canCD1A6, canCD1A8) revealed a complete sequence without obvious characteristics of pseudogenes like internal stop codons, frameshift mutations, and splice site mutations. Five CD1A genes were considered to be pseudogenes (canCD1A1, canCD1A3, canCD1A4, canCD1A5, canCD1A7). During the in silico study, we found a fourth full-length CD1A gene; however, due to its high nucleotide identity with canCD1A8 (93\%) and because it was not assigned to chromosome 38 by Ensemble (CanFam 2.0, www.ensembl.org), we assume that this is an allelic variation and named these two $\mathrm{CD} 1 \mathrm{~A} 8$ genes canCD1A8.1 


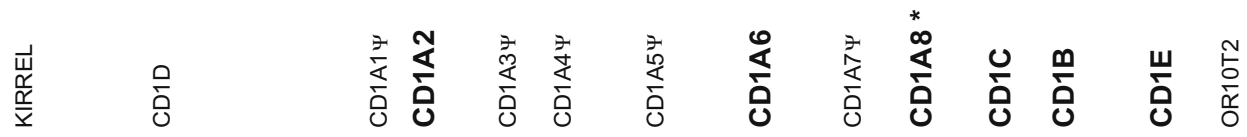

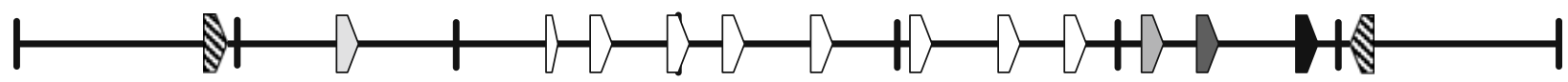 \\ $\begin{array}{llll}26.60 \mathrm{Mbp} & 26.55 & 26.50 & 26.45\end{array}$ \\ Fig. 1 Complete gene map of the canine CD1 locus located on chromosome 38. Full-length canine CD1 genes without characteristics of pseudogenes are given in bold. Pseudogenes are marked with $\Psi$. Exact location of CD1 genes: CD1D: unknown-26502267; CD1A1: 26479022-26477829; CD1A2: 26470445-26467658; CD1A3: 26452485-26449619; CD1A4: 26439630-26436644; CD1A5: \\ 26.40 \\ 26.35 \\ 26.30 \\ 26.25 Mbp \\ 26418868-26416544; CD1A6: 26398237-26395316; CD1A7: 26378486-26375657; CD1A8: 26363063-26360148; CD1B: 26332684-26329120; CD1C: 26344187-26341100; CD1E: 26310804-26308062. Asterisk, The location of the allelic variant of CD1A8 is: $81854142-81857071$ on an unknown chromosome (Ensemble)}

and canCD1A8.2, accordingly. Furthermore, full-length genes for canCD1B, canCD1C, and canCD1E were present in the dog CD1 locus. In the draft sequence (AC183576), we found a part of the canine CD1D gene. In this sequence, it was not possible to identify the leader fragment, the first intron, and a small part of the $\alpha 1$ domain of canCD1D because the nucleotide sequence was unknown, but most of the $\alpha 1$ domain and the complete $3^{\prime}$ part of the canCD1D gene were intact. Because the map of the locus is complete in the sense that the locus between the adjacent non-CD1 Kirrel and olfactory receptor genes did not contain any gaps big enough to contain a CD1 gene, we assume that we identified all canCD1 genes.

\section{Cloning of full-length canCD1 cDNAs}

Full-length cDNAs were cloned using a PCR-based strategy. The forward primer was designed to anneal upstream of the start codon, based on the available genomic sequences, and the reverse primer was designed to bind downstream of the predicted stop codon. Full-length canCD1A6 (GenBank EU373508/protein_id ACB12088) and canCD1A8.2 (GenBank EU373509/protein_id ACB12089) transcripts were cloned. We have not been able to obtain full-length CD1A2, which may be due to a low transcription level in thymus cDNA and high homology with canCD1A8 at the sites where the primers were designed to bind. The ectodomain of the canCD1A6 transcript was $97 \%$ identical at the amino acid level to the predicted canCD1A6 transcript from the in silico study. The canCD1A8 transcript showed greater amino acid identity to the predicted canCD1A8.2 transcript than to the predicted canCD1A8.1 transcript (98\% and $88 \%$, respectively). A single full-length canCD1B transcript was cloned (GenBank EU373510/protein id ACB12090) and showed $99 \%$ amino acid identity with the predicted canCD1B. Two different full-length canCD1C transcripts were obtained from thymus-derived cDNA of a single dog (Beagle), which was named $\operatorname{dog} \mathrm{A}$. The two different transcripts canCD1C clone 1 and clone 2 (GenBank
EU373511/protein_id ACB12091 and EU373512/protein_id ACB12092) both showed $98 \%$ amino acid identity to the predicted canCD1C transcript. These small nucleotide alterations compared to the predicted sequence might be caused by PCR artifacts and/or allelic variation. A third unique fulllength canCD1C, clone 3 (GenBank EU373513/protein id ACB12093), was obtained from cDNA derived from the 030 D canine histiocytosis cell line and showed $99 \%$ identity with the predicted canCD1C. From thymus-derived cDNA, a partial canCD1D transcript was cloned containing a fragment of the $\alpha 2$ domain, full-length $\alpha 3$ domain, a transmembrane region, and a cytoplasmic tail.

Recognition of canCD1 molecules by monoclonal antibodies

To determine which antibodies recognize the canine CD1 isoforms, we tested a panel of mAbs specific for CD1 in humans, cattle, sheep, and cat. We also tested the two anticanine CD1 mAbs CA13.9H11 and CA9.AG5 for their specificity for canCD1. Results are summarized in Table 1. Freshly isolated thymocytes of dog A were recognized by the anti-canine mAb CA13.9H11 and CA9.AG5, the anti human $\mathrm{mAb} \mathrm{BCD} 1 \mathrm{~b} 3$, the anti-bovine $\mathrm{mAb} \mathrm{CC} 20$ and SBUT6, and the anti-feline mAb Fe1.5F4 and $\mathrm{Fe} 5.5 \mathrm{C} 1$ but not by the anti-human CD1a mAb OKT-6 and anti-human CD1c mAb F10/21A3. Using 293T cells transfected with full-length canCD1 transcripts, we found that canCD1a6 is recognized by the anti-feline $\mathrm{CD} 1 \mathrm{a} \mathrm{Fe} 1.5 \mathrm{~F} 4$ but not by anticanine CD1 CA13.9H11. The opposite was true for the recognition of canCD1a8.2 (Fig. 2). This implies that two different $\mathrm{mAbs}$ recognize two different in vivo expressed canCD1a proteins. The anti-canine CD1 antibody CA9.AG5 did not recognize canCD1a6, canCD1a8.2, and canCD1b protein on transfected $293 \mathrm{~T}$ cells; however, it did recognize an unidentified epitope on canine thymocytes.

293T cells transfected with the full-length canCD1C transcript clone 1 and 2, derived from $\operatorname{dog} \mathrm{A}$, or canCD1C transcript clone 3 , derived from the 030-D cell line, were 
Table 1 A panel of anti-CD1 mAb was tested for recognition of canCD1 on transfectant 293T cells, canine thymocytes, and 030-D canine histiocytosis cell line

\begin{tabular}{|c|c|c|c|c|c|c|c|}
\hline $\mathrm{mAb}$ & Canine thymocytes & 030-D & canCD1a6 & canCD1a8.2 & canCD1b & Published reactivity & Reference \\
\hline CA13.9H11 & + & + & - & + & - & canCD1 & Moore et al. 1996 \\
\hline CA9.AG5 & + & - & - & - & - & canCD1 & Moore et al. 1996 \\
\hline WM-35 & - & + & - & - & - & huCD1a & \\
\hline BCD1b3 & + & + & - & + & + & huCD1b & \\
\hline SBUT6 & + & $+/-$ & - & + & + & boCD1a/boCD1b3 & Mackay et al. 1985 \\
\hline $\mathrm{CC} 20$ & + & + & - & + & - & huCD1b/boCD1b3 & Howard et al. 1993 \\
\hline $\mathrm{Fe} 1.5 \mathrm{~F} 4$ & + & - & + & - & - & felCD1a & Woo and Moore 1997 \\
\hline $\mathrm{Fe} 5.5 \mathrm{C} 1$ & + & + & - & - & - & felCD1c & Woo and Moore 1997 \\
\hline
\end{tabular}

not recognized by any of the mAbs in this panel. Unfortunately, we have not been able to confirm expression of the three full-length canCD1c cDNAs by staining with any $\mathrm{mAb}$ or by alternative methods. The mAbs CA13.9H11 and CA9.AG5 have both been suggested to recognize canCD1 (Moore et al. 1996). In this study, we show that CA13.9H11 recognizes canCD1a8.2. We have not been able to show CD1 recognition by CA9.AG5. The mAb CA9.AG5 recognizes a molecule expressed on thymocytes of approximately $60 \%$ of all dogs, suggesting that this $\mathrm{mAb}$ is specific for an allotype or allelic variation of an unidentified gene product. In our study, the same animal (dog A) was used for flowcytometric analysis of ex vivo thymocytes and for cDNA synthesis from thymocytes. The ex vivo thymocytes of this animal were recognized by CA9.AG5, but the two slightly different CD1c cDNAs were not. This makes it unlikely that the lack of recognition by the anti-canine CD1 antibodies CA13.9H11 and CA9.AG5 is caused by allelic differences in canCD1c.

Transcription of CD1A genes in different tissues

Transcription levels of the three different CD1A genes were determined in different tissues of a healthy Beagle. The
Fig. 2 Flow cytometric analysis of expression canCD1 on tranfectant 293 T cells. Cells are transfected with canCD1A6 $(\mathbf{a}-\mathbf{b})$ or with canCD1A8.2 gene transcripts (c-d). Staining was performed with anti-canine CD1 $\mathrm{mAb} \mathrm{CA} 13.9 \mathrm{H} 11$ and with antifeline $\mathrm{CD} 1 \mathrm{a} \mathrm{mAb}$, followed by GAM-FITC

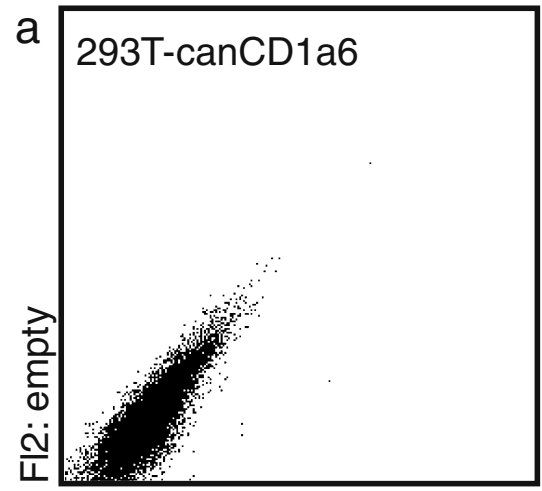

FI1: CA13.9H11-gamFITC

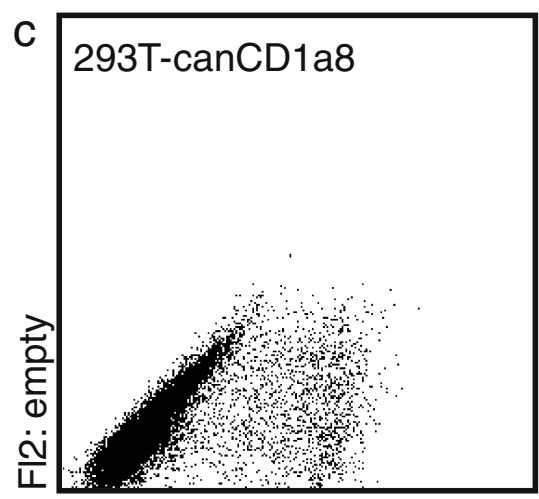

Fl1: CA13.9H11-gamFITC

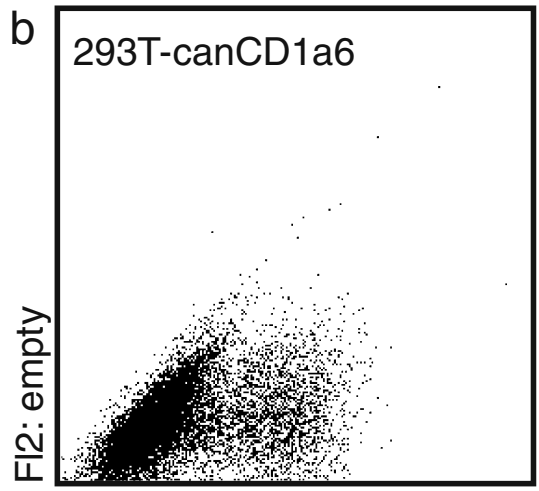

Fl1: Fe1.5F4-gamFITC

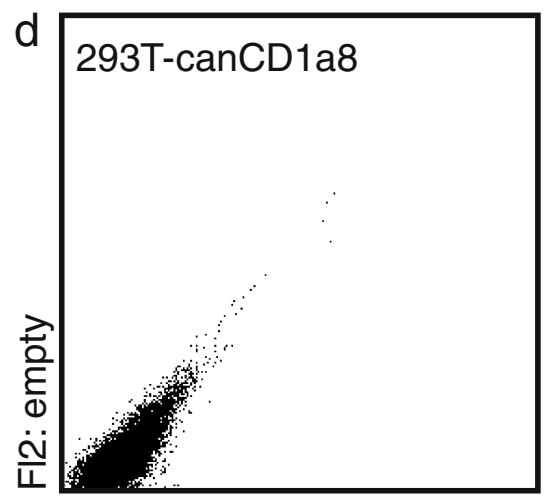

Fl1: Fe1.5F4-gamFITC 
expression of different CD1A genes in canine lymphoid and nonlymphoid tissues is shown in Fig. 3. The transcription of canCD1A2 was only found in the thymus, whereas canCD1A6 and canCD1A8 were transcribed in the thymus, lymph node, spleen, liver, skin, and PBMCs. It is interesting to note that, in skin, canCD1A8 is clearly expressed at a much higher level than the other CD1A genes. Results are representative of three independent experiments.

Comparison of canCD1a6 and canCD1a8 models and canine CD1 cytoplasmic tails

The protein sequence of the two different canCD1a molecules of which a full-length transcript was derived is very similar. However, single amino acid differences at key positions in the binding groove can result in noticeable differences in the structure of the binding groove and, moreover, in the ability to bind and present lipids. To predict whether the overall architecture of the antigenbinding grooves of canCD1a is similar to that of human CD1a, we generated models of the two in vivo expressed canCD1a molecules using the Swiss Model server (Schwede et al. 2003) and compared these to their human counterpart (Fig. 4a). Both the structural models and the sequence comparison (Fig. 4b) suggest that both canCD1a6 and canCD1a8, like human CD1a (Zajonc et al. 2005), have a hydrophobic binding groove with a buried $\mathrm{A}^{\prime}$ pocket and a more exposed $\mathrm{F}^{\prime}$ pocket. Key structural features such as the $\mathrm{A}^{\prime}$ pole are conserved (Phe70 and Val/Ile12), as well as an $\mathrm{A}^{\prime}$ roof is predicted (residues 66, 69, 161, 165). In addition, the $\mathrm{A}^{\prime}$ pocket of canCD1a8 is predicted to be open ended (Gly28 vs. Val or Ala), similar to mouse CD1d. Therefore, the length of bound lipids in that pocket could be longer than $\mathrm{C}_{18}$, as observed for human CD1a (Zajonc et al. 2003). Clearly the shape of the individual pockets will be slightly different, as not all groove-lining residues are conserved. However, the overall size of the CD1a grooves appears to be similar. Furthermore, the groove opening of canCD1a8 appears slightly larger than the opening of canCD1a6. Furthermore, the residues lining the opening to the $\mathrm{CD} 1 \mathrm{a}$ groove $\left(\mathrm{F}^{\prime}\right.$ portal) are the least conserved between the CD1a molecules. As these residues were shown to bind the glycolipid headgroup, we speculate that the three CD1a proteins have the capacity to bind different glycolipid antigens.

Furthermore, different antigens are presented by CD1 proteins due to sampling in different endocytic compartments. This intracellular trafficking of CD1 is directed by the presence or absence of a sorting motif in the cytoplasmic tail. We aligned cytoplasmic tail sequences of the three canine CD1a proteins with the closely related carnivore species cat and to other, more distantly related mammalian species (Fig. 5a). To make this alignment, we performed a blast search in the cat genome and found four CD1A genes of which two contained the nucleotide sequence of the cytoplasmic tail, and we used known CD1a cytoplasmic tail sequences of human (NM001763), rabbit (AF276977 and AF276978), pig (AF059492), and cattle (DQ192541). Except for human CD1a and canCD1a8.2, all other known mammalian CD1a cytoplasmic tails are long. CanCD1a2 and canCD1a8.1 have an even

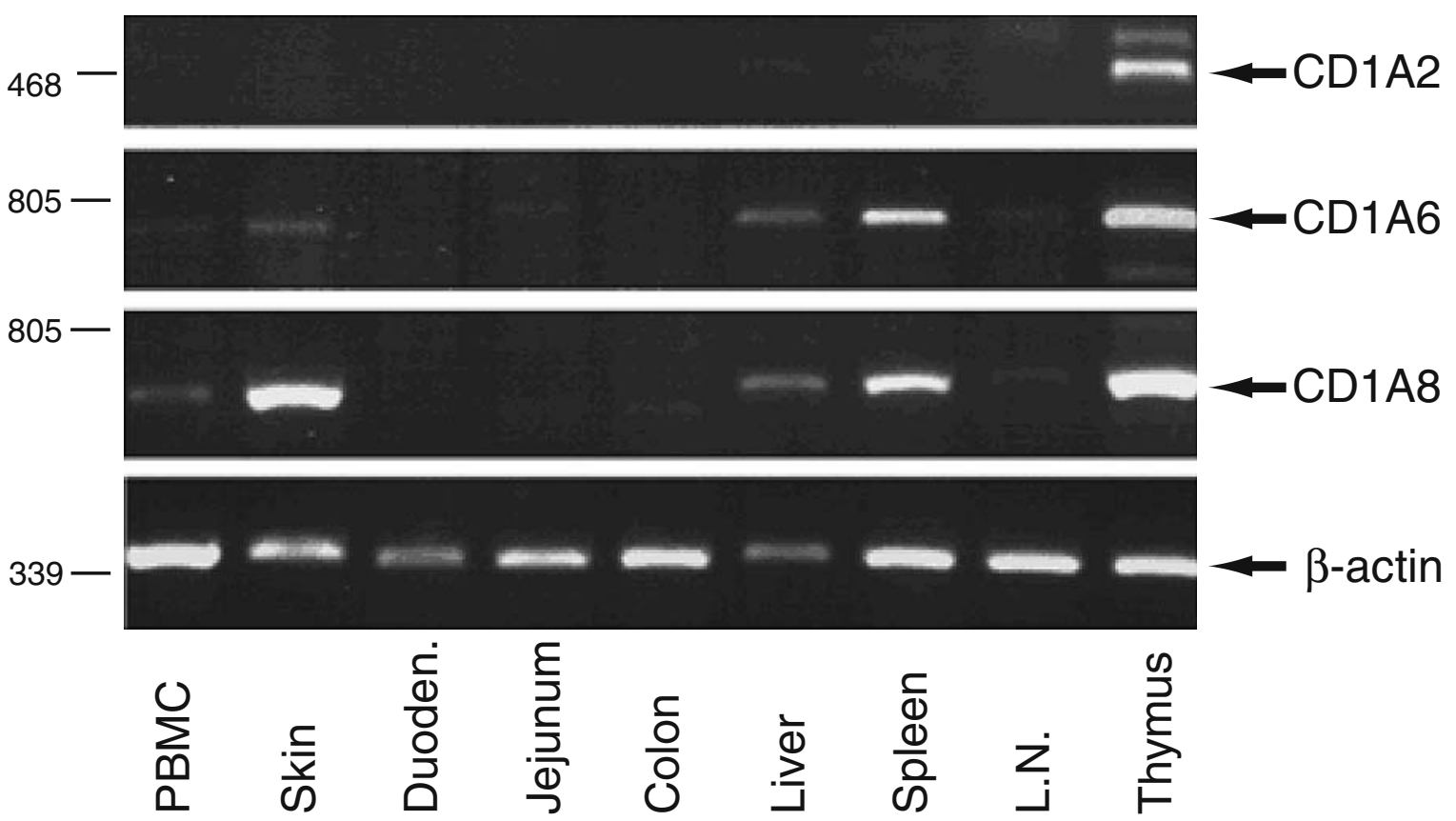

Fig. 3 Transcription of three canCD1A genes in different dog tissues. To be able to compare the quantity of input cDNA, $\beta$-actin was included 
a

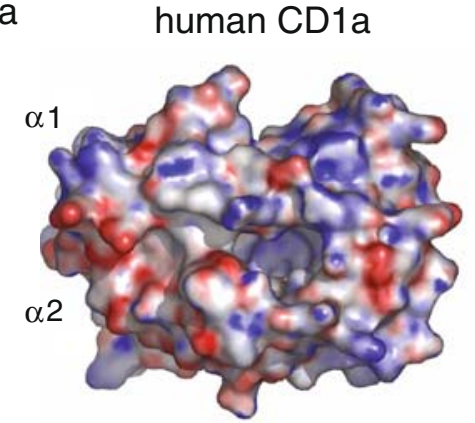

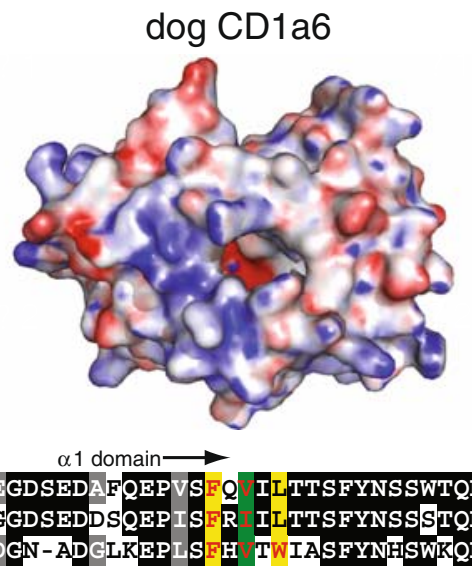

R

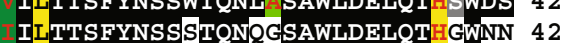

canCDIa8

Leader $\longrightarrow$ humanCD1a

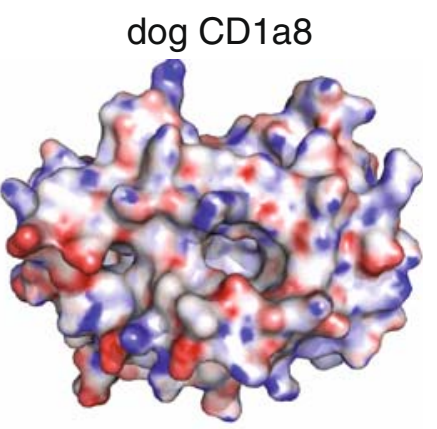

b

$\alpha 2$ domain $\longrightarrow$

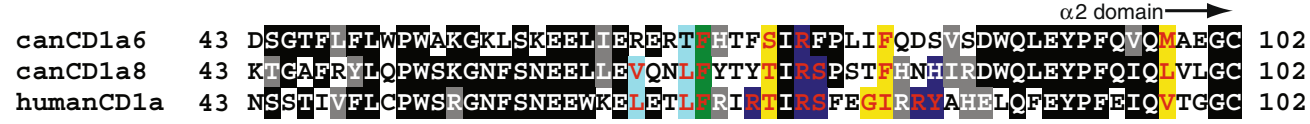

canCD1a6 103 GLYFGKPSVGFMQIAYQGSDLVSFQNKSWWPSPKGGRRAQVVCKLLNQYHVVÑLRIHSHI 162 canCD1a8 103 DSHFGEASAGFLLAYQGSDVLSFQNT SWRPSPEGGSRAQKVCSLFNQDHVSH IVRKLI 162 humanCD1a 103 ELHSGKVSGSELQAYQGSDFVFQNNSWLPYPVA GNMAKHFCKVLNQ QHENDITHNLL 162

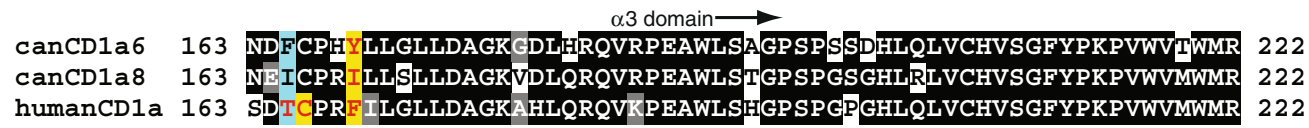
humanCD1a 163 SDTCPRFILGLLDAGKAHLOROVK PEAWLSHGPSPGPGHLOLVCHVSGFYPKPVWVW
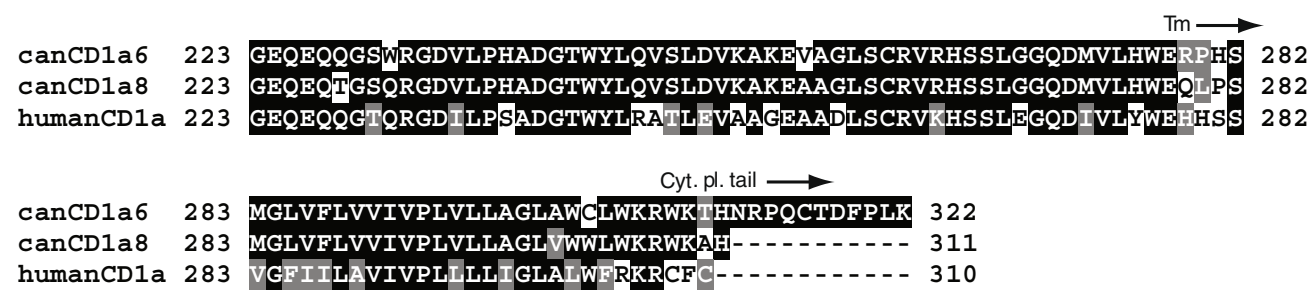

Fig. 4 a Molecular surface of human CD1a, canine CD1a6, and canine CD1a8. The $\alpha 1$ and $\alpha 2$ domains are depicted, and the opening to the binding groove is in the center of each molecule. Note that the bottom of the canCD1a6 binding groove appears electronegative due to the presence of Glu100; however, overall, the binding grove is hydrophobic. Furthermore, residue 100 is not highlighted in Fig. 5b, as it was not a residue contacting the sulfatide ligand in the human CD1a-sulfatide structure (1onq). Electrostatic potentials were calculated using APBS and PyMol; red is electronegative, and blue is electropositive $(-30$ to $+30 \mathrm{kT} / \mathrm{e})$. b Multiple-sequence alignment (CLUSTAL W 1.83) of canine CD1a isoforms 6 and 8 and human

longer cytoplasmic tail of 31 amino acids, which was confirmed by the identification of transcripts of canCD1A8.1 (DN877612) and canCD1A2 (DN409579) that included these cytoplasmic tails in the EST databases. No known sorting motif was observed in any of the canine CD1a cytoplasmic tail sequences.

The cytoplasmic tails of the other canine CD1 isoforms were aligned with the amino acid sequences of cytoplasmic tails of their human homologs (Fig. 5b). Like in their human counterparts, the tyrosine trafficking motif YXXZ was present in all three canine sequences. However, the
CD1a, colored by sequence identity (black) and structural features (in color). Residues contributing to the CD1a binding groove of human $\mathrm{CD} 1 \mathrm{a}$ are highlighted in yellow. These residues were originally identified base on their interaction with the sulfatide ligand (PDB code 1onq). Darkgreen, $\mathrm{A}^{\prime}$ pole; green, terminal residue restricting the length of the A' pocket; cyan, $\mathrm{A}^{\prime}$ roof; blue, residues at the opening to the CD1a groove ( $\mathrm{F}^{\prime}$ portal). Residues colored in red or gray are similar. Residues that are highlighted but not colored in red are predicted not to dramatically alter the structural feature. Canine residues not highlighted according to their human counterparts are predicted to have an altered structural element

human CD1c tail contains a dileucine motif, which is not present in canCD1c.

\section{Discussion}

The canine CD1 locus located on chromosome 38 contained all known CD1 isoforms. We identified a remarkable large number of $\mathrm{CD} 1 \mathrm{~A}$ homologs, three of which were shown to be full-length canCD1A genes and five were considered pseudogenes. Intensive duplication of 


humanCD1a
cancD1a8.2
canCD1a2
canCD1a8.1
canCD1a6
felcD1a1 (p)
felcD1a2 (p)
rabbitCD1a1
rabbitCD1a2
pigCD1.1
bocD1a

CFC

WKAH

WKAHWRPQCMDFPSEQEPSSPSSSTYLNPAQH

WKAHWRPQCTDFPSEQEPSSPGSSTYLNPAQH WKTHNRPQCTDFPLK

WKSHETPQCTGLPLE

WKSHWRHQCTGLPLE

WIHHG. PLETLLPLQ

WSHHGSPN . SLLPLK

WK. HCDPSSALHRLE

WTHRESPSS . VLPLE

Fig. 5 a Alignment of the CD1a cytoplasmic tail sequences of different mammalian species. Tail sequences that were not confirmed by cDNA sequencing but only predicted from the genome are marked

CD1A genes leading to the presence of two or possibly three distinct CD1a proteins is characteristic for the canine CD1 locus. This is the first study showing differential transcription of two CD1A genes for which in vivo protein expression is confirmed. These differences in expression might indicate differences in function between the CD1a molecules in canine skin. So far, it is unknown whether these different CD1a molecules are present on the same antigenpresenting cell in the canine skin or that different antigenpresenting cells express different canCD1a molecules. Stationary epidermal Langerhans cells as well as migrating dermal Langerhans cells have a high CD1a expression. Besides Langerhans cells, also a subpopulation of dermal dendritic cells has been reported to express CD1a (Angel et al. 2006). These professional antigen-presenting cells play an important role in the initiation of the immune response and are able to activate T cells in a CD1a-restricted manner (Pena-Cruz et al. 2003; Kissenpfennig et al. 2005).

Increased numbers of $\mathrm{CD} 1 \mathrm{c}^{+}$Langerhans cells have been described in lesional skin of dogs with atopic dermatitis (Olivry et al. 1996, 1997, 2006). In these two studies, the primary monoclonal antibody CA13.9H11 was used to detect canCD1c. However, from our study using 293T cells transfected with the different CD1 isoforms, we know that CA13.9H11 recognizes canCD1a8.2. It is possible that CA13.9H11 recognizes both CD1a8.2 and canCD1c, but we have not been able to demonstrate recognition of canCD1c by this $\mathrm{mAb}$ so far. Therefore, it is possible that the reported expression of $\mathrm{CD} 1$ on Langerhans cells in lesional canine skin is reflecting expression of canCD1a8 rather than canCD1c. The current assignment of canCD1c being the molecule recognized by anti-canine $\mathrm{CA} 13.9 \mathrm{H} 11$ has not been invalidated in this study. However, 293T cells transfected with three different full-length canCD1C transcripts were not recognized by CA13.9H11. The mAb CA9.AG5 has been used to determine canCD1a expression on Langerhans cells (Olivry et al. 1996). In our study, $293 \mathrm{~T}$ cells transfected with canCD1A6, canCD1A8.2, canCD1B, or the three canCD1C sequences were not recognized by CA9.AG5. It is possible that the mAb CA9.AG5 does not recognize canCD1 but an unknown epitope on canine thymocytes.

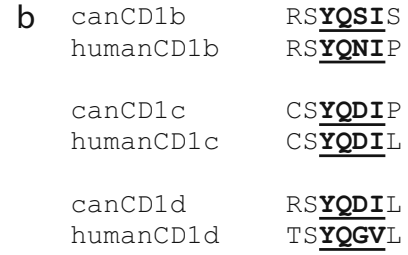

accordingly $(p)$. b Alignment of cytoplasmic tail sequences of canCD1b, canCD1c, and canCD1d with their human orthologs. The tyrosine trafficking motif (YXXZ) is underlined

The current study shows the expression of two different types of canine CD1a proteins. We found differences in the ectodomain between the two molecules and the unique presence of two different cytoplasmic tails, which might contribute to a greater scope of glycolipid antigen presentation and improved skin immunity.

Rabbits are the only other mammalian species that are known to have two different CD1a proteins (Hayes and Knight 2001). However, cytoplasmic tails of the two rabbit CD1a proteins show higher sequence identity compared to dogs. Based on the presence of long cytoplasmic tails in all mammalian species other than primates, it is likely that the canCD1A gene encoding a short cytoplasmic tail (canCD1A8.2) is derived from a paralog with a long cytoplasmic tail. Single-nucleotide point mutations may have introduced a stop codon within the long tail sequence resulting in a shorter tail. Indeed, the 31-amino acid-long cytoplasmic tails of canCD1a2 and canCD1a8.1 can be changed into the short, four-amino acid-long cytoplasmic tail like the one in CD1a8.2, by a single point mutation. The cytoplasmic tail of the human CD1a ortholog does not contain any known endosomal targeting sequences (Sugita et al. 2007). However, whether this also holds for CD1a molecules of other mammalian species and whether they traffic like their human counterpart are unknown. Like their human homologs, a tyrosine motif was present in the cytoplasmic tail of canCD1b and canCD1d. This might indicate that these canine CD1 molecules traffic to similar compartments as human $\mathrm{CD} 1 \mathrm{~b}$ and human $\mathrm{CD} 1 \mathrm{~d}$ compartments to sample lipid antigens. Canine CD1c also contained a tyrosine-trafficking motif within its cytoplasmic tail, but unlike human $\mathrm{CD} 1 \mathrm{c}$, a dileucine motif was not present.

CD1 gene duplication might be driven by the lack of certain $\mathrm{CD} 1$ isoforms. One of the four CD1B genes found in guinea pig seems to replace the loss of a guinea pig CD1A gene (Hiromatsu et al. 2002). The extensive canine CD1A isoform duplication found in this study seems not to be driven by the limited repertoire of other CD1 isoforms because all isoforms are present within the canine CD1 locus. This suggests that for canCD1A, gene duplication 
was driven by another reason than the replacement of loss of other CD1 isoforms.

The presence of multiple CD1A genes in dogs, their differential transcription in healthy skin, and upregulation at least for canCD1a8 during allergic skin diseases (Olivry et al. 1996 ; 1997) in combination with their potential to present different glycolipid antigens suggest different functions in skin immunity.

Acknowledgments We thank H. van Engelen and A. van der Lee for their technical assistance. The financial support for this study was provided by Royal Canin, France.

Declaration I declare that all experiments in this study comply with the current laws of the country in which the experiments were performed.

Open Access This article is distributed under the terms of the Creative Commons Attribution Noncommercial License which permits any noncommercial use, distribution, and reproduction in any medium, provided the original author(s) and source are credited.

\section{References}

Angel CE, George E, Brooks AE, Ostrovsky LL, Brown TL, Dunbar PR (2006) Cutting edge: CD1a+ antigen-presenting cells in human dermis respond rapidly to CCR7 ligands. J Immunol 176:5730-5734

Baker NA, Sept D, Joseph S, Holst MJ, McCammon JA (2001) Electrostatics of nanosystems: application to microtubules and the ribosome. Proc Natl Acad Sci USA 98:10037-10041

Barral DC, Brenner MB (2007) CD1 antigen presentation: how it works. Nat Rev Immunol 7:929-941

Beckman EM, Porcelli SA, Morita CT, Behar SM, Furlong ST, Brenner MB (1994) Recognition of a lipid antigen by CD1restricted alpha beta+ T cells. Nature 372:691-694

Beckman EM, Melian A, Behar SM, Sieling PA, Chatterjee D, Furlong ST, Matsumoto R, Rosat JP, Modlin RL, Porcelli SA (1996) CD1c restricts responses of mycobacteria-specific T cells. Evidence for antigen presentation by a second member of the human CD1 family. J Immunol 157:2795-2803

Bendelac A, Lantz O, Quimby ME, Yewdell JW, Bennink JR, Brutkiewicz RR (1995) CD1 recognition by mouse NK1+ T lymphocytes. Science 268:863-865

Brigl M, Brenner MB (2004) CD1: antigen presentation and T cell function. Annu Rev Immunol 22:817-890

Briken V, Jackman RM, Watts GF, Rogers RA, Porcelli SA (2000) Human CD1b and CD1c isoforms survey different intracellular compartments for the presentation of microbial lipid antigens. J Exp Med 192:281-288

Calabi F, Milstein C (1986) A novel family of human major histocompatibility complex-related genes not mapping to chromosome 6. Nature 323:540-543

Chen YH, Chiu NM, Mandal M, Wang N, Wang CR (1997) Impaired $\mathrm{NK} 1+\mathrm{T}$ cell development and early IL-4 production in CD1deficient mice. Immunity 6:459-467

Dascher CC, Hiromatsu K, Naylor JW, Brauer PP, Brown KA, Storey JR, Behar SM, Kawasaki ES, Porcelli SA, Brenner MB, LeClair
KP (1999) Conservation of a CD1 multigene family in the guinea pig. J Immunol 163:5478-5488

De Libero G, Mori L (2005) Recognition of lipid antigens by T cells. Nat Rev Immunol 5:485-496

Dougan SK, Kaser A, Blumberg RS (2007) CD1 expression on antigen-presenting cells. Curr Top Microbiol Immunol 314:113141

Ernst WA, Maher J, Cho S, Niazi KR, Chatterjee D, Moody DB, Besra GS, Watanabe Y, Jensen PE, Porcelli SA, Kronenberg M, Modlin RL (1998) Molecular interaction of CD1b with lipoglycan antigens. Immunity 8:331-340

Gumperz JE, Miyake S, Yamamura T, Brenner MB (2002) Functionally distinct subsets of CD1d-restricted natural killer $\mathrm{T}$ cells revealed by CD1d tetramer staining. J Exp Med 195: 625-636

Hayes SM, Knight KL (2001) Group 1 CD1 genes in rabbit. J Immunol 166:403-410

Hiromatsu K, Dascher CC, Sugita M, Gingrich-Baker C, Behar SM, LeClair KP, Brenner MB, Porcelli SA (2002) Characterization of guinea-pig group $1 \mathrm{CD} 1$ proteins. Immunology 106:159-172

Howard CJ, Sopp P, Bembridge G, Young J, Parsons KR (1993) Comparison of CD1 monoclonal antibodies on bovine cells and tissues. Vet Immunol Immunopathol 39:77-83

Jackman RM, Stenger S, Lee A, Moody DB, Rogers RA, Niazi KR, Sugita M, Modlin RL, Peters PJ, Porcelli SA (1998) The tyrosine-containing cytoplasmic tail of $\mathrm{CD} 1 \mathrm{~b}$ is essential for its efficient presentation of bacterial lipid antigens. Immunity 8:341351

Kawano T, Cui J, Koezuka Y, Toura I, Kaneko Y, Motoki K, Ueno H, Nakagawa R, Sato H, Kondo E, Koseki H, Taniguchi M (1997) CD1d-restricted and TCR-mediated activation of valpha14 NKT cells by glycosylceramides. Science 278:1626-1629

Kissenpfennig A, Henri S, Dubois B, Laplace-Builhe C, Perrin P, Romani N, Tripp CH, Douillard P, Leserman L, Kaiserlian D, Saeland S, Davoust J, Malissen B (2005) Dynamics and function of Langerhans cells in vivo: dermal dendritic cells colonize lymph node areas distinct from slower migrating Langerhans cells. Immunity 22:643-654

Levy MG, Gebhard D, Gager R, Breitschwerdt EB (1995) A newly described canine monocytoid cell line capable of supporting growth of the rickettsial parasite Ehrlichia canis, and useful for examination of host:parasite interactions (abstract). In: Proceedings of the International Union of Immunology Society, 4th International Veterinary Immunology Symposium, p 274

Mackay CR, Maddox JF, Gogolin-Ewens KJ, Brandon MR (1985) Characterization of two sheep lymphocyte differentiation antigens, SBU-T1 and SBU-T6. Immunology 55:729-737

Moody DB, Reinhold BB, Guy MR, Beckman EM, Frederique DE, Furlong ST, Ye S, Reinhold VN, Sieling PA, Modlin RL, Besra GS, Porcelli SA (1997) Structural requirements for glycolipid antigen recognition by CD1b-restricted T cells. Science 278:283286

Moody DB, Ulrichs T, Muhlecker W, Young DC, Gurcha SS, Grant E, Rosat JP, Brenner MB, Costello CE, Besra GS, Porcelli SA (2000) CD1c-mediated T-cell recognition of isoprenoid glycolipids in Mycobacterium tuberculosis infection. Nature 404:884888

Moody DB, Young DC, Cheng TY, Rosat JP, Roura-Mir C, O'Connor PB, Zajonc DM, Walz A, Miller MJ, Levery SB, Wilson IA, Costello CE, Brenner MB (2004) T cell activation by lipopeptide antigens. Science 303:527-531

Moore PF, Schrenzel MD, Affolter VK, Olivry T, Naydan D (1996) Canine cutaneous histiocytoma is an epidermotropic Langerhans cell histiocytosis that expresses CD1 and specific beta 2-integrin molecules. Am J Pathol 148:1699-1708 
Niazi K, Chiu M, Mendoza R, Degano M, Khurana S, Moody D, Melian A, Wilson I, Kronenberg M, Porcelli S, Modlin R (2001) The $\mathrm{A} \phi$ and $\mathrm{F} \phi$ pockets of human $\mathrm{CD} 1 \mathrm{~b}$ are both required for optimal presentation of lipid antigens to $\mathrm{T}$ cells. J Immunol $166: 2562-2570$

Olivry T, Moore PF, Affolter VK, Naydan DK (1996) Langerhans cell hyperplasia and $\operatorname{IgE}$ expression in canine atopic dermatitis. Arch Dermatol Res 288:579-585

Olivry T, Naydan DK, Moore PF (1997) Characterization of the cutaneous inflammatory infiltrate in canine atopic dermatitis. Am J Dermatopathol 19:477-486

Olivry T, Deangelo KB, Dunston SM, Clarke KB, McCall CA (2006) Patch testing of experimentally sensitized beagle dogs: development of a model for skin lesions of atopic dermatitis. Vet Dermatol 17:95-102

Pena-Cruz V, Ito S, Dascher CC, Brenner MB, Sugita M (2003) Epidermal Langerhans cells efficiently mediate CDla-dependent presentation of microbial lipid antigens to $\mathrm{T}$ cells. J Invest Dermatol 121:517-521

Schwede T, Kopp J, Guex N, Peitsch MC (2003) SWISS-MODEL: an automated protein homology-modeling server. Nucleic Acids Res 31:3381-3385

Shamshiev A, Gober HJ, Donda A, Mazorra Z, Mori L, De Libero G (2002) Presentation of the same glycolipid by different CD1 molecules. J Exp Med 195:1013-1021

Shiina T, Ando A, Suto Y, Kasai F, Shigenari A, Takishima N, Kikkawa E, Iwata K, Kuwano Y, Kitamura Y, Matsuzawa Y, Sano K, Nogami M, Kawata H, Li S, Fukuzumi Y, Yamazaki M,
Tashiro H, Tamiya G, Kohda A, Okumura K, Ikemura T, Soeda E, Mizuki N, Kimura M, Bahram S, Inoko H (2001) Genomic anatomy of a premier major histocompatibility complex paralogous region on chromosome 1q21-q22. Genome Res 11:789-802

Spada FM, Koezuka Y, Porcelli SA (1998) CD1d-restricted recognition of synthetic glycolipid antigens by human natural killer $\mathrm{T}$ cells. J Exp Med 188:1529-1534

Sugita M, Jackman RM, van Donselaar E, Behar SM, Rogers RA, Peters PJ, Brenner MB, Porcelli SA (1996) Cytoplasmic taildependent localization of $\mathrm{CD} 1 \mathrm{~b}$ antigen-presenting molecules to MIICs. Science 273:349-352

Sugita M, Grant EP, van Donselaar E, Hsu VW, Rogers RA, Peters PJ, Brenner MB (1999) Separate pathways for antigen presentation by $\mathrm{CD} 1$ molecules. Immunity 11:743-752

Sugita M, Barral DC, Brenner MB (2007) Pathways of CD1 and lipid antigen delivery, trafficking, processing, loading, and presentation. Curr Top Microbiol Immunol 314:143-164

Woo JC, Moore PF (1997) A feline homologue of CD1 is defined using a feline-specific monoclonal antibody. Tissue Antigens 49:244-251

Zajonc DM, Elsliger MA, Teyton L, Wilson IA (2003) Crystal structure of CD1a in complex with a sulfatide self antigen at a resolution of $2.15 \mathrm{~A}$. Nat Immunol 4:808-815

Zajonc DM, Crispin MD, Bowden TA, Young DC, Cheng TY, Hu J, Costello CE, Rudd PM, Dwek RA, Miller MJ, Brenner MB, Moody DB, Wilson IA (2005) Molecular mechanism of lipopeptide presentation by CD1a. Immunity 22:209-219 